\title{
Safe management of laparoscopic endoscopic cooperative surgery for superficial non-ampullary duodenal epithelial tumors $\square$
}

다(1)우우

\author{
Authors \\ Takeshi Azuma², Yoshihiro Kakeji ${ }^{1}$ \\ Institutions \\ 1 Division of Gastrointestinal Surgery, Department of \\ Surgery, Kobe University Graduate School of Medicine, \\ Kobe, Japan \\ 2 Division of Gastrointestinal Medicine, Department of \\ Internal Medicine, Kobe University Graduate School of \\ Medicine, Kobe, Japan
}

Yasunori Otowa ${ }^{1}$, Shingo Kanaji ${ }^{1}$, Yoshinori Morita², Satoshi Suzuki ${ }^{1}$, Masashi Yamamoto ${ }^{1}$, Yoshiko Matsuda ${ }^{1}$, Takeru Matsuda ${ }^{1}$, Taro Oshikiri ${ }^{1}$, Tetsu Nakamura' ${ }^{1}$, Fumiaki Kawara², Shinwa Tanaka², Tsukasa Ishida², Takashi Toyonaga²,

submitted 11.4.2017

accepted after revision 3.7.2017

Bibliography

DOI https://doi.org/10.1055/s-0043-117957 |

Endoscopy International Open 2017; 05: E1153-E1158

(c) Georg Thieme Verlag KG Stuttgart · New York

ISSN 2364-3722

Corresponding author

Yasunori Otowa, Division of Gastrointestinal Surgery, Department of Surgery, Kobe University Graduate School of Medicine, Kusunoki-chou 7-5-2, Chuo-ku, Kobe 650-0017, Japan

Fax: +81-78-382-5939

otoway@med.kobe-u.ac.jp

\section{ABSTRACT}

Background and study aims Endoscopic submucosal dissection (ESD) for duodenal tumors results in a high delayed perforation rate due to the thinness of the duodenal wall. In most cases with perforation after duodenal ESD, additional surgery is needed due to severe peritonitis. A newly developed procedure, laparoscopic endoscopic cooperative surgery for duodenal tumors (D-LECS), may help to avoid perforation after ESD. In our institution, patients with superficial non-ampullary duodenal epithelial tumors (SNADET) smaller than $50 \mathrm{~mm}$ which could not have en-bloc resection by endoscopic mucosal resection were treated with DLECS. After a laparoscopic exposure of anterior duodenal wall of second portion, ESD was performed. Laparoscopic suturing from the serosal side of ESD site was performed for reinforcement. There were neither postoperative leakage nor other complications. Therefore, D-LECS can be performed safely and prevent perforation after ESD for SNADET. D-LECS could be selected as a treatment for SNADET which can be cured by ESD.

\section{Introduction}

Despite the fact that duodenal cancer is still very rare, recent improvements in endoscopy using high magnification for observing mucosal patterns and network vascular patterns have contributed to the early detection and accurate diagnosis of tumor invasion [1]. Because lymphadenectomy is not required for duodenal intramucosal carcinoma given the very low rate of lymph node metastasis [2,3], endoscopic treatment is recommended to avoid invasive procedures. Endoscopic submucosal dissection (ESD) is reported to have a higher en-bloc resection rate than endoscopic mucosal resection (EMR) [4, 5]. However, when ESD is performed in the duodenum, there is a high risk of complications such as perforation and bleeding (6-50\% and
$0-7 \%$, respectively) due to the thinness of the duodenal wall and technical difficulties in narrow duodenal lumen [6, 7]. Further, duodenal delayed perforation was reported in some cases even after ESD without intraoperative perforation [6, 8].

Recently, laparoscopic endoscopic cooperative surgery for duodenal tumors (D-LECS) has been developed as a new procedure which can prevent perforation by reinforcing the ESD site. However, details of the operative procedure and tips for performing D-LECS remain unclear. Herein, we report the procedural methods for performing D-LECS, which resulted in no postoperative perforation. 
- Table 1 Therapeutic indications for D-LECS.

1. Patients with SNADET

2. SNADET that is smaller than $50 \mathrm{~mm}$ and is not suitable for en-bloc resection by EMR

3. Patients without past history of upper abdominal surgery

SNADET, superficial non-ampullary duodenal epithelial tumors; EMR, endoscopic mucosal resection

\section{Patients and methods}

\section{Patients}

Ten patients from September 2014 to November 2016 who had a duodenal tumor and were suitable for treatment by ESD were retrospectively enrolled in the study. All patients underwent diagnostic endoscopy with biopsy. Our therapeutic indications for D-LECS are shown in - Table 1. EMR is applied to SNADET when it is smaller than $10 \mathrm{~mm}$. All study participants provided informed consent, and the study design was approved by the ethics review board at Kobe University Hospital and conformed to the provisions of the 1995 Declaration of Helsinki.

\section{Operative technique}

\section{Patient positioning}

Under general anesthesia, the operation was started with the patient in the left lower half lateral decubitus position. When the surgeon performed the laparoscopic procedure, the operating table was rotated to the right side so that the patient was in a supine position. When the endoscopist performed the ESD, the operating table was rotated to the left side so the patient was in the left lateral decubitus position ( $\vee$ Fig. $1 \mathbf{a}$ ).

\section{Laparoscopic Kocher maneuver}

The operator stood on the patient's left side. The trocars were

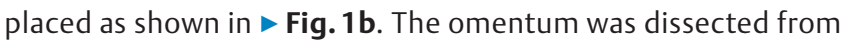
the right lateral side up to the front of the duodenum and the mesocolon was removed from the epigastric tissue. The procedure continued with dissection of the right hepatocolic ligament, with ultrasonic coagulating shears (SonoSurg, Olympus Medical System, Tokyo, Japan) being used to mobilize the right colic flexure. When the anterior wall of the duodenal second portion was well exposed, the laparoscopic Kocher maneuver was stopped to prevent difficulties with ESD due to loss of duodenal fixation on the retroperitoneum ( $\triangleright$ Video 1 ). After the ESD, the surgeon moved to the right side of the patient and completed the Kocher maneuver beyond the vena cava until the ESD site was well exposed.

\section{Endoscopic submucosal dissection}

First, endoscopic markings were made on the mucosa surrounding the lesion after spraying indigo carmine using a forward-viewing endoscope (for example, GIF-Q260]; Olympus Medical System Co., Tokyo, Japan) and a Flush knife BT $1.5 \mathrm{~mm}$ (Fujifilm Medical Co., Tokyo, Japan). Sodium hyaluronate solu-

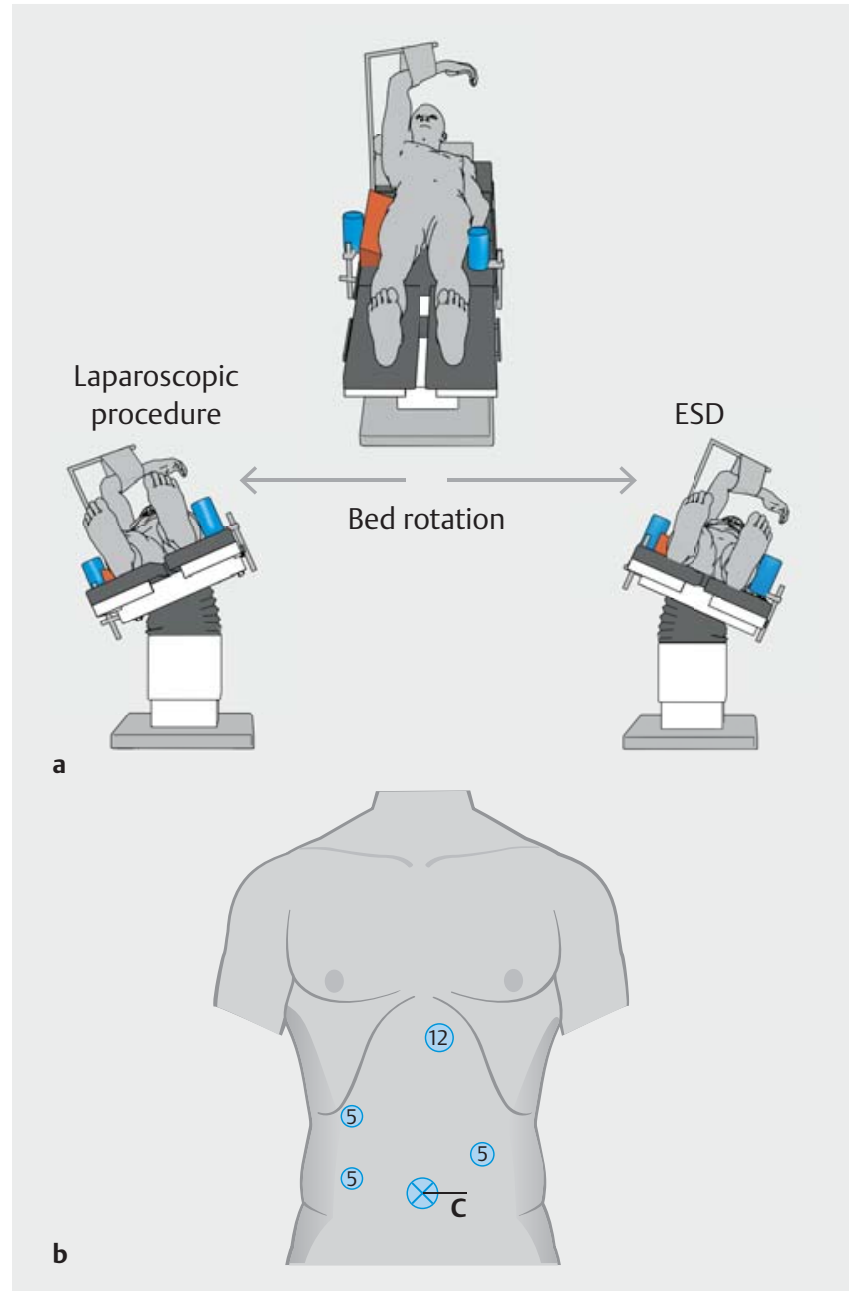

Fig. 1 a The patient is placed in the left lower half lateral decubitus position at the beginning of the operation. The operating table is rotated to the right side to move the patient into the supine position for the laparoscopic procedure and left side to move the patient into the left lateral decubitus position for the endoscopic procedure. b A blunt trocar is inserted from the umbilicus. A $12-\mathrm{mm}$ trocar and $35-\mathrm{mm}$ trocars are inserted as indicated.

tion (0.4\%) (MucoUp; Seikagaku Corp., Tokyo, Japan) was injected into the submucosal layer before the initial mucosal incision. After circumferential mucosal incision using a Flush knife BT $1.5 \mathrm{~mm}$, a clip with thread was placed at the oral edge of the target lesion. Adequate traction was obtained by pulling the thread gently. Clip traction and frequent additional injections using the knife were effective and safe during submucosal dissection. A Flush knife BT was used with a short ST hood (DH28GR; Fujifilm Medical Co., Tokyo, Japan) for dissection. In some cases, a Clutch Cutter (Fujifilm Medical Co., Tokyo, Japan) was also used in order to avoid perforation ( $\triangleright$ Video 2 ).

\section{Laparoscopic suturing}

The ESD site was easily identified from the serosal side due to white coloration caused by thermal denaturation ( $>$ Fig. 2a). In addition, laparoscopic identification of the ESD site from the serosal side was possible in all patients by observing the trans- 

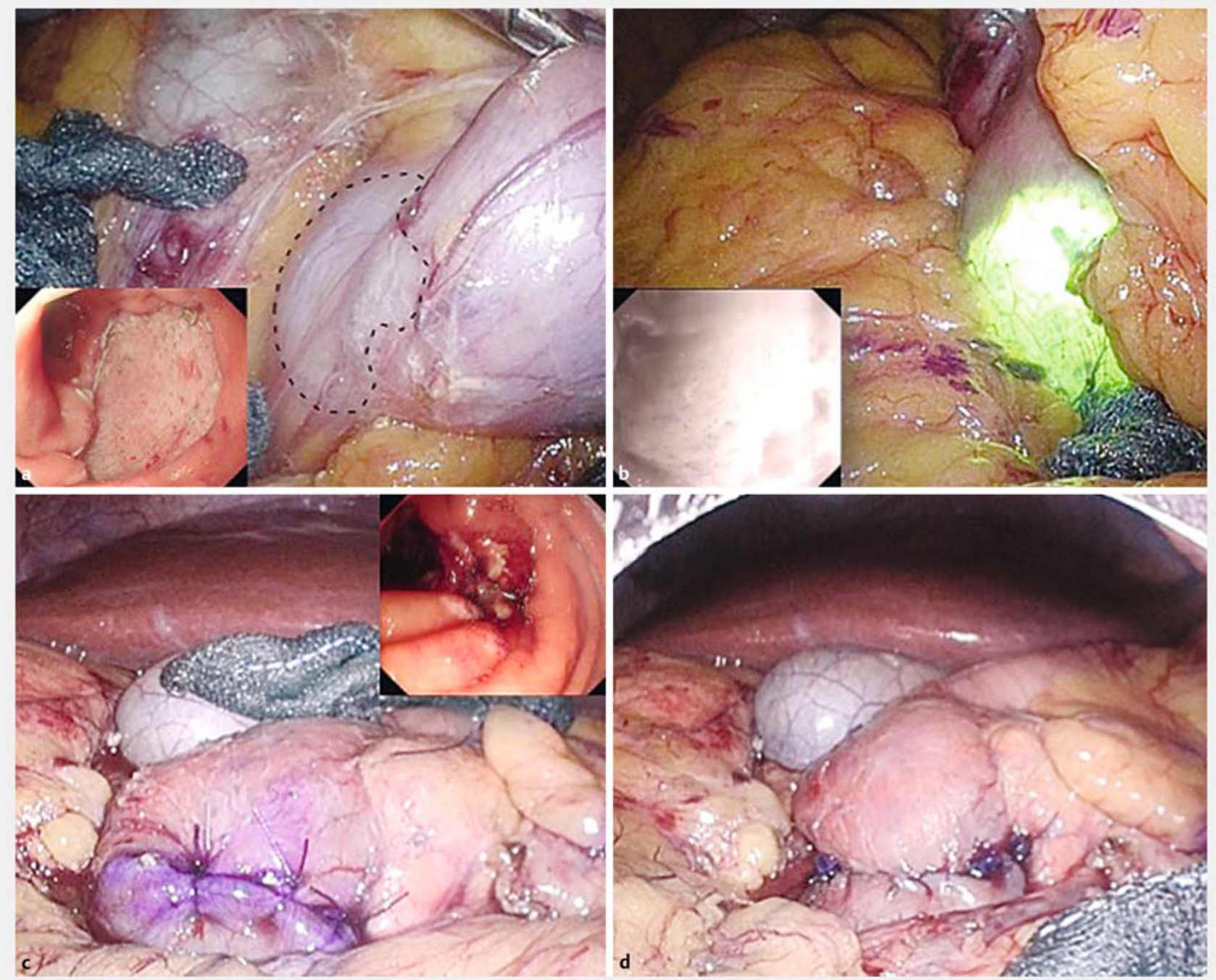

- Fig. 2 a Laparoscopic view and endoscopic view of the endoscopic submucosal dissection site (dotted line). b The endoscopically dissected sited is identified from the transmitted light of the endoscope. c Laparoscopic manual intracorporeal knotted suture in the full thickness layer of the duodenal wall was carried out followed by confirmation by endoscopy. $\mathbf{d}$ Laparoscopic running suture in the seromuscular layer of the duodenal wall was carried out and then the re-endoscopic observation was performed.

mitted light of the endoscope ( $\bullet$ Fig.2b). Two-layer suturing was carried out laparoscopically even when no perforation was suspected. Laparoscopic suturing of the duodenal wall was carried out with a manual intracorporeal knotted suture using a 3-0 vicryl suture (Ethicon GMbH, Norderstedt, Germany) in the full thickness layer. After the suturing, the endoscopic observation was performed to confirm the closure of ESD site ( $\boldsymbol{F}$ Fig. $\mathbf{2 c}$ ). If inadequate, additional suturing with full-thickness layer was performed. Then, running suture using a 3-0 PDS ${ }^{\mathrm{TM}}$ (Ethicon) with Lapra-Ty ${ }^{\mathrm{TM}}$ (Ethicon) clips in the seromuscular layer was performed. After the suturing of the duodenal wall, the re-endoscopic observation was performed to confirm a patent lumen, any leakage and bleeding ( $\bullet$ Fig. $\mathbf{2 d}$, $\checkmark$ Video 3$)$.

\section{Outcomes}

Data on postoperative leakage rate within 1 month after surgery, laparotomy conversion rate, surgery-related death and any postoperative complications more than grade II according to the Clavien-Dindo classification of surgical complications [9] were collected to assess the safety of D-LECS.

\section{Results}

Patient characteristics and surgical outcomes are shown in - Table 2. The median age was 63.5 years, and there were 7 male patients. All tumors were located in the second portion and 3 were located above ampulla and 7 were located bellow ampulla. Two patients were preoperatively diagnosed with cancer. The median laparoscopy procedure time was 120.5 min- 
utes and the ESD procedure time was 99.5 minutes. Four patients had a perforation during ESD; however, no patients had postoperative leakage including delayed perforation. No patients had any other complications and there were no surgery-related deaths. The median size of resected specimen was $37 \mathrm{~mm}$. The median times of starting food intake and discharge from hospital were the fourth and eleventh postoperative days, respectively. All dissected specimens were histologically found to be cancer with negative margins. All patients were able to maintain their food intake without any restriction.

\section{Discussion}

Simple laparoscopic resection may be a less invasive alternative for duodenal tumors. However, for SNADET, it is difficult to determine the resection line with adequate margin from the serosal surface of laparoscopic view even using endoscopic view. Laparoscopic excessive resection may cause deformity or/and stenosis and insufficient surgical margins may result in local recurrence. Adequate maneuver of the duodenum is necessary for suturing the duodenal ESD site; however, suturing from small wound including laparoscopic assisted surgery is difficult, especially for posterior side of duodenum. Considering invasiveness and maneuverability, reinforcement using technique of D-LECS is valid option for safe management after duodenal ESD.

However, there are some technical problems with D-LECS. First, suitable patient positioning differs between an endoscopic and a laparoscopic procedure. Generally, the supine position is suitable for laparoscopic procedures, whereas the left lateral decubitus position is suitable for endoscopic procedures. To overcome this problem, we started the operation in the left lower half lateral decubitus position. This position allows the patient to be moved into both the supine and left lat-

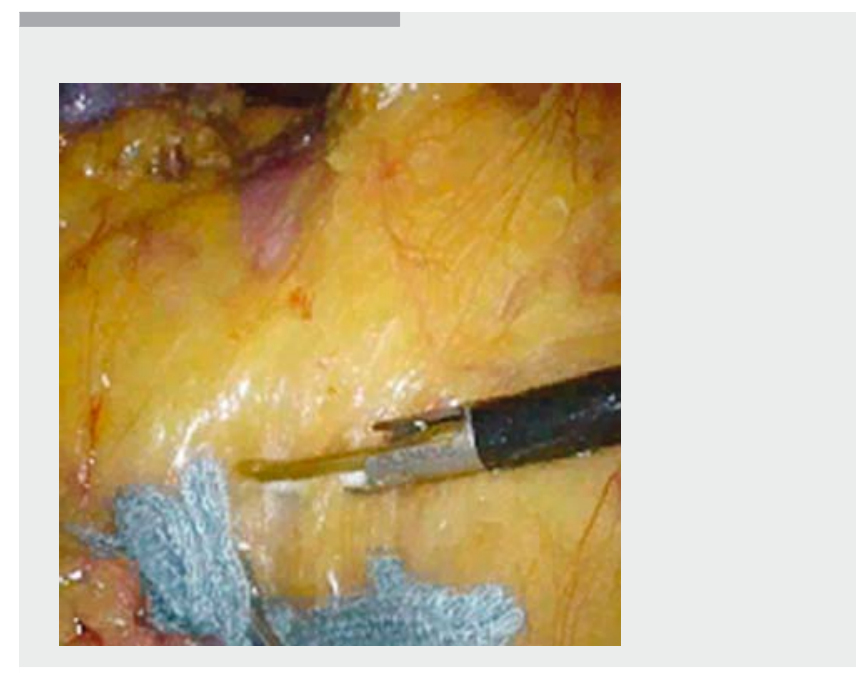

Video 1 The omentum was dissected and the mesocolon was removed from the epigastric tissue. Right hepatocolic ligament was dissected to mobilize the right colic flexure. Kocher maneuver was stopped after exposing the anterior and right sides of the duodenal second portion. eral decubitus positions that are suitable for laparoscopic and endoscopic procedures. Another technical problem is the endoscopic maneuverability, which is reduced due to loss of duodenal fixation on the retroperitoneum after the Kocher maneuver. However, the laparoscopic Kocher maneuver after ESD makes it difficult to recognize the proper layer due to leakage of $\mathrm{CO}_{2}$ during ESD. Therefore, to retain endoscopic maneuverability, the Kocher maneuver was stopped until exposure of the anterior wall of the second part of the duodenum. After completion of the ESD procedure, the Kocher maneuver was completed by exposing the posterior side of the second part of the duodenum, if necessary.

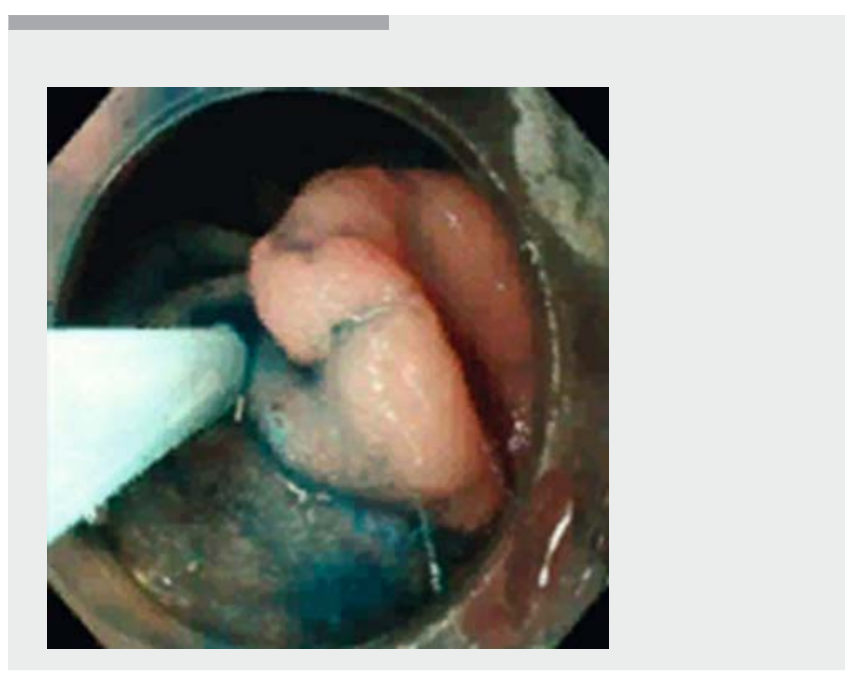

Video 2 Endoscopic markings followed by circumferential mucosal incision was performed. Then a clip with thread was placed at the oral edge of the lesion for an adequate counter traction. Clip traction and additional injections using the knife were effective during dissection.

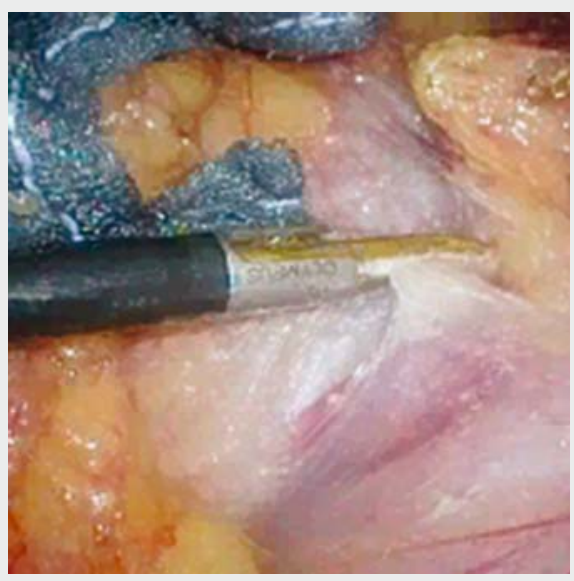

Video 3 Kocher maneuver was performed until the endoscopic submucosal dissection (ESD) site was well exposed. The ESD site was identified by the transmitted light of the endoscope and then two-layer suturing of the duodenal wall was carried out. 
- Table 2 Patient characteristics and operative outcomes

\section{D-LECS $(n=10)$}

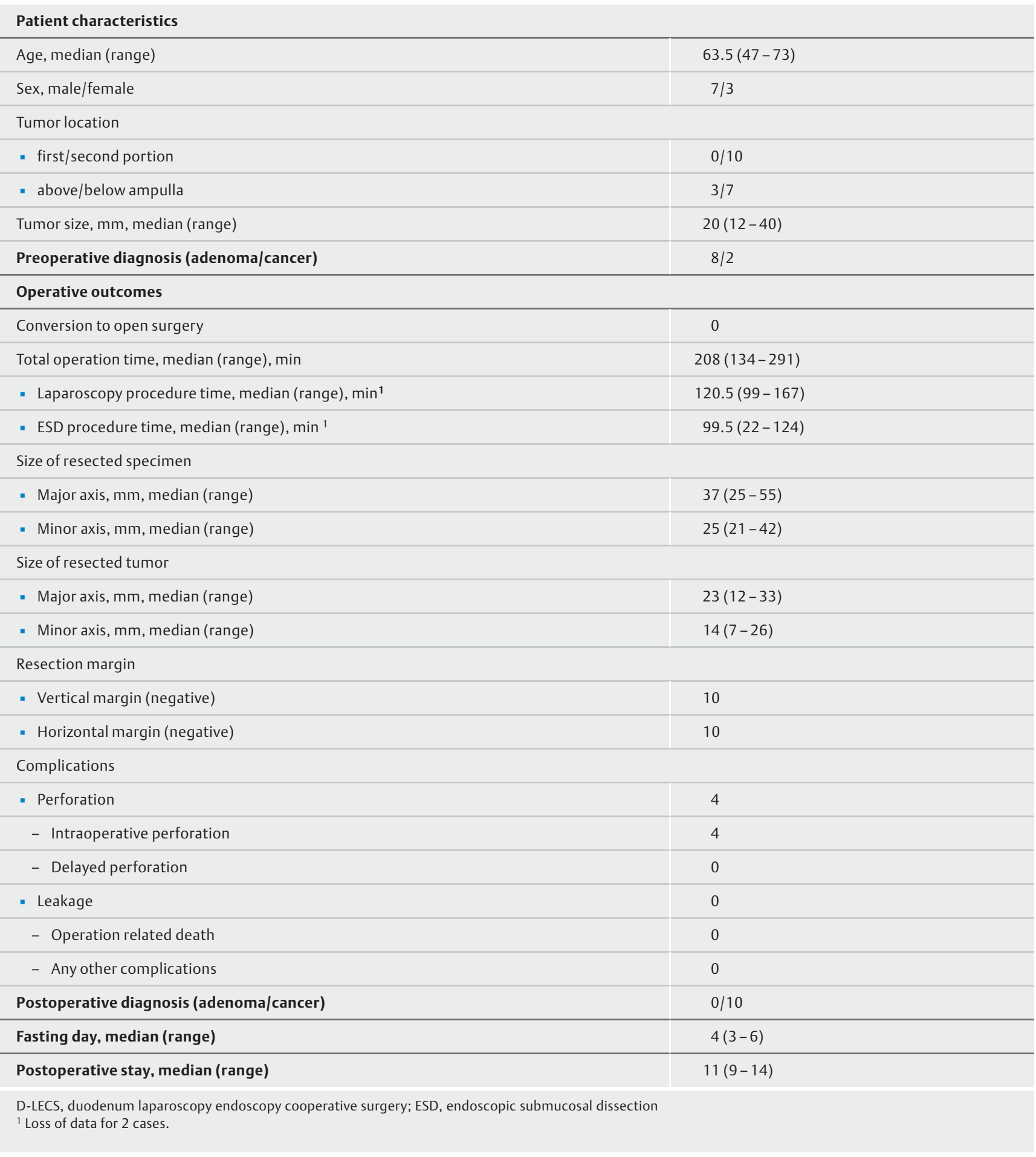

In our early experience, $50 \%$ of cases had a perforation after treating SANDETS with ESD alone (data not shown), while no patients had perforation when treated with D-LCES. Therefore, ensuring confirmation and reinforcement of thin duodenal wall after ESD is essential for the laparoscopic procedure during D-LECS. The transmitted light from the endoscope is useful for laparoscopic detection of the thin duodenal wall after ESD. Further, confirmed closure of ESD site after suturing is necessary for secure prevention of delayed perforation. We believe that rough closure of the ESD site by suturing the full-thickness layer prior to suturing the seromuscular layer is useful for secure reinforcement of the whole ESD site. Further, reinforcement by 
suturing the full-thickness layer may contribute to prevention of postoperative bleeding.

\section{Conclusion}

Despite including a small number of patients due to the low incidence of duodenal tumors, our report is important as it demonstrates a surgical technique for avoiding perforation after duodenal ESD. All patients in the current study had no complications and were able to maintain their food intake. We were able to achieve en-bloc resection by ESD in cases in which the ESD site was near the Vater's ampulla and large circumferential lesions; however, further study is needed to determine the technical limitations of D-LECS. Because all of the duodenal tumors were intramucosal carcinoma, strict follow-up and examination of long-term outcomes are necessary.

\section{Competing interests}

Dr. Toyonaga invented the Flush Knife-BT in conjunction with Fujifilm and received royalties from its sale.

\section{References}

[1] Yoshimura N, Goda K, Tajiri H et al. Endoscopic features of nonampullary duodenal tumors with narrow-band imaging. Hepatogastroenterology 2010; 57: $285-289$

[2] Nagatani K, Takekoshi T, Baba Y et al. Indications for endoscopic treatment of early duodenal cancer. Based on cases reported in the literature. Endosc Digest 1993; 5: 969-976 (in Japanese with an English abstract)

[3] Fujisawa T, Tomofuji Y, Kuroda $\mathrm{N}$ et al. A case of duodenal cancer with tubule-villous adenoma: Report of a case and clinicopathological review of Japanese literature. Gastroenterol Endosc 1995; 37: 2768 2775 (in Japanese with an English abstract)

[4] Matsumoto S, Miyatani H, Yoshida Y. Endoscopic submucosal dissection for duodenal tumors: a single-center experience. Endoscopy 2013; 45: $136-137$

[5] Jung JH, Choi KD, Ahn JY et al. Endoscopic submucosal dissection for sessile, nonampullary duodenal adenomas. Endoscopy 2013; 45: $133-135$

[6] Kakushima N, Kanemoto H, Tanaka M et al. Treatment for superficial non-ampullary duodenal epithelial tumors. World J Gastroenterol 2014; 20: $12501-12508$

[7] Yamamoto Y, Yoshizawa N, Tomida H et al. Therapeutic outcomes of endoscopic resection for superficial non-ampullary duodenal tumor. Dig Endosc 2014; 26: (Suppl. 02): 50-56

[8] Marques J, Baldaque-Silvia F, Pereira P et al. Endoscopic mucosal resection and endoscopic submucosal dissection in the treatment of sporadic nonampullary duodenal adenomatous polyps. World J Gastrointest Endosc 2015; 7: $720-727$

[9] Dindo D, Demartines N, Clavien PA. Classification of surgical complications: a new proposal with evaluation in a cohort of 6336 patients and results of a survey. Ann Surg 2004; 240: 205-213 